Pacific Journal of Mathematics

AN ANALOGUE OF KOLMOGOROV'S THREE-SERIES
THEOREM FOR ABSTRACT RANDOM VARIABLES 


\title{
AN ANALOGUE OF KOLMOGOROV'S THREE-SERIES THEOREM FOR ABSTRACT RANDOM VARIABLES
}

\author{
R. P. PAKShIRAJAN
}

1. Introduction. $(\Omega, \angle \mathscr{K}, P)$ is a probability space i.e. $\Omega$ is an abstract set of points $w, \mathscr{C}$ is a $\sigma$-field of subsets of $\Omega$ and $P$ is a nonnegative countably additive set function defined on $\mathscr{C}$ such that $P(\Omega)=1$. $G$ is a locally compact Hausdorff abelian metric topological group. The group operation in $G$, as well as in the several other groups to be dealt with, will be denoted by + . Let $e$ denote the identity element of $G$. By the Borel sets of $G$ we mean the sets belonging to the $\sigma$ ring generated by the class $\mathscr{C}$ of compact subsets of $G$. Let $\mathscr{D}$ be the class of subsets of $G$ whose intersection with every compact set is a Borel set. Notice that $\mathscr{D}$ is a $\sigma$-field containing the open subsets of $G$. The character group of $G$ will be denoted by $\hat{G}$. A single valued mapping $f$ of $\Omega$ into $G$ will be called a generalised random variable (g.r.v.) if $f^{-1}(A) \in \mathscr{C l}$ whenever $A \in \mathscr{D}$. An immediate consequence of this definition is that if $f$ is a g.r.v. then $\eta(f)$ is an ordinary (complex valued) random variable for every $\eta \in \hat{G}$. A finite or a countably infinite collection of g.r.v.'s is said to be independent if and only if for every finite subset $\left\{X_{i}, i=1,2, \cdots, n\right\}$ of distinct members of the collection and for every choice of sets $A_{j} \in \mathscr{D}, j=$ $1,2, \cdots, n$ it is true that $P\left\{w: X_{i}(w) \in A_{i}, i=1,2, \cdots, n\right\}=\prod_{1}^{n} P\{w$ : $\left.X_{i}(w) \in A_{i}\right\}$.

If $G$ is the real line, $\hat{G}$ is the real line too. For $t \in \hat{G}$ and $x \in G$, $t(x)=\exp (i t x)$. Given the random variable $X$ and any real number $c>0$ we define a new random variable $Y=t_{0} \alpha$ where $t_{0}=c / \pi$ and $\alpha$ is the principal amplitude of $\exp (i \pi X / c)$. The two sets $\{w:-c<X(w) \leqq c\}$ and $\{w: X(w) \neq Y(w)\}$ are then seen to be equal. Denoting by $N$ the interval $(-c, c]$, the classical three series theorem [2] may be stated thus: If $\left\{X_{n}, n=1,2, \cdots\right\}$ is a sequence of independent real valued random variables then $\sum_{1}^{\infty} X_{n}$ exists with probability 1 (a.e.) if and only if, for some $c>0$, the following three series converge.

(i) $\sum_{1}^{\infty} P\left\{w: X_{n}(w) \notin N\right\}$

(ii) $\sum_{1}^{\infty} E Y_{n}$ and

(iii) $\sum_{1}^{\infty} \operatorname{var} Y_{n}$.

$E$ and var denote respectively the mathematical expectation and

Received August 1962. This research was supported in part by funds provided under contract No. DA-O4-200-ORD-651 with the office of ordnance Research, U.S. Army and was carried out under the guidance of Professsor Herman Rubin at the University of Oregon, U.S.A. 
variance. $\alpha_{n}$ is the principal amplitude of $\exp \left(i \pi X_{n} / c\right)$ and $Y_{n}=t_{0} \alpha_{n}$. The convergence of the above three series is easily seen to be equivalent to the convergence of

(i) $\quad \sum_{1}^{\infty} P\left\{w: X_{n}(w) \notin N\right\}$

(ii) $\sum_{1}^{\infty} E \log t\left(X_{n}\right)$ and

(iii) $\sum_{1}^{\infty} \operatorname{var} \log t\left(X_{n}\right)$ for every $t \in \hat{G}, \log t\left(X_{n}\right)$ being defined to be equal to $i \theta_{n}$ where $\theta_{n}$ is the principal amplitude of $\exp \left(i t X_{n}\right)$. It is in this form the classical three series theorem lends itself for extension to the case of generalised random variables. In $\S 2$ three lemmas are proved leading to the generalisation. In $\S 3$ we give a neccessary and sufficient condition for the convergence almost every where of $\sum_{1}^{\infty} X_{n}$ in terms only of characters and not using characterstic functions.

The following two known results are quoted for the sake of completeness and ready reference.

THEOREM A. (Cor. (2.1) [4]).

If $\left\{h_{n}, n=1,2, \cdots\right\}$ is a sequence of continuous homomorphisms on a topological group $G_{1}$ to a toplogical group $G_{2}$ which converge pointwise to $h$ throughout some Baire set of the second category then $h$ is continuous.

\section{Theorem B. (\$2.21 [3]).}

Let $G$ be a locally compact abelian group. Let $N$ be a compact symmetric neighbourhood of $e$. Let $G^{\prime}$ be the subgroup of $G$ generated by $N$. Then $G^{\prime}$ contains a discrete subgroup $D$ with a finite number of generators such that the quotient group $G^{\prime} / D$ is compact and $D \cap(N+N+N)=\{e\}$.

2. For a sequence of real or complex numbers $g_{n}, n=1,2, \cdots$ we say that $\Pi_{1}^{\infty} g_{n}$ exists if $\Pi_{n}^{\infty} g_{k}$ is nonzero for sufficiently large $n$.

Lemma 1. For $\eta \in \hat{G}$, a necessary and sufficient condition that $\Pi_{1}^{\infty} \eta\left(X_{n}\right)$ exists a.e. is that $\Pi_{1}^{\infty} E \eta\left(X_{n}\right)$ exists.

Proof. If $\Pi_{1}^{\infty} \eta\left(X_{n}\right)$ exists a.e. then, by the bounded convergence theorem, $\Pi_{1}^{\infty} \operatorname{E\eta }\left(X_{n}\right)$ exists.

Conversely let $\Pi_{1}^{\infty} E \eta\left(X_{n}\right)$ exist. Hence $\Pi_{1}^{\infty}\left|E \eta\left(X_{n}\right)\right|$ exists. Let $\eta\left(X_{n}(w)\right)=\exp \left(i \theta_{n}(w)\right)$ where $\theta_{n}(w)$ is the principal value of the amplitude. Hence $\theta_{1}, \theta_{2}, \cdots$ is a bounded, independent sequence of real valued random variables. Let $\theta_{n}^{\prime}$ be the symmetrised version of $\theta_{n}$ and let $\theta_{n}^{\prime}$ (1) be $\theta_{n}^{\prime}$ truncated at 1 . One has (p. 196, [2]) var $\theta_{n}^{\prime}$ $(1) \leqq 3\left\{1-\left|E \eta\left(X_{n}\right)\right|^{2}\right\}$. Hence $\sum_{1}^{\infty} \operatorname{var} \theta_{n}^{\prime}(1)<\infty$. By the classical three series theorem it follows that $\sum_{1}^{\infty} \theta_{n}^{\prime}$ converges a.e. Consequently (p. 250, [2]) there exist constants $\alpha_{n}$ such that $\sum_{1}^{\infty}\left(\theta_{n}-\alpha_{n}\right)$ exists 
a.e. or equivalently $\Pi_{1}^{\infty} \exp \left(-i \alpha_{n}\right) E \eta\left(X_{n}\right)$ exists. This implies the convergence of $\sum_{1}^{\infty} \alpha_{n}$ since $\Pi_{1}^{\infty} E \eta\left(X_{n}\right)$ is assumed to converge. We now conclude $\sum_{1}^{\infty} \theta_{n}$ exists a.e. or, what is same, $\Pi_{1}^{\infty} \eta\left(X_{n}\right)$ exists a.e.

Lemma 2. For a given $\eta \in \widehat{G}$, the following two sets of conditions are equivalent.

$$
\begin{aligned}
& \prod_{1}^{\infty} E \eta\left(X_{n}\right) \text { exists; } \sum_{1}^{\infty} \operatorname{var} \eta\left(X_{n}\right)<\infty \\
& \sum_{1}^{\infty} E \theta_{n} \text { converges; } \sum_{1}^{\infty} \operatorname{var} \theta_{n}<\infty
\end{aligned}
$$

where $\eta\left(X_{n}\right)=\exp \left(i \theta_{n}\right), \theta_{n}$ being the principal amplitude.

Proof. Suppose (2.2) holds. Therefore by the three series theorem on the line, $\sum_{1}^{\infty} \theta_{n}$ exists a.e. This implies that $\Pi_{1}^{\infty} \eta\left(X_{n}\right)$ exists a.e. Hence $\prod_{1}^{\infty} E \eta\left(X_{n}\right)$ exists by the bounded convergence.

Let now $\alpha_{n}=E \theta_{n} ; \beta_{n}=\operatorname{var} \theta_{n}$ and $\theta_{n}=\alpha_{n}+y_{n}$. As in the last lemma, $E \eta\left(X_{n}\right)=\left(1+d_{n} \beta_{n} / 2\right) \exp \left(i \alpha_{n}\right)$ where $\left|d_{n}\right| \leqq 1$.

$$
\begin{aligned}
E\left|\eta\left(X_{n}\right)-E \eta\left(X_{n}\right)\right|^{2} & =E\left|\exp \left(i y_{n}\right)-\left(1+d_{n} \beta_{n} / 2\right)\right|^{2} \\
& \leqq c \beta_{n} \text { where } c \text { is an absolute constant } \\
& =c \operatorname{var} \theta_{n} .
\end{aligned}
$$

Hence $\sum_{1}^{\infty} \operatorname{var} \eta\left(X_{n}\right)<\infty$.

Conversely, suppose (2.1) holds.

$$
\begin{aligned}
\operatorname{var} \eta\left(X_{n}\right) & =E\left|\exp \left(i y_{n}\right)-\left(1+d_{n} \beta_{n} / 2\right)\right|^{2} \\
& =1+\left|1+d_{n} \beta_{n} / 2\right|^{2}-2 \text { real part of } E\left(\overline{1+d_{n} \beta_{n} / 2}\right) \exp \left(i y_{n}\right) \\
& =1-\left|1+d_{n} \beta_{n} / 2\right|^{2} .
\end{aligned}
$$

Hence $\sum_{1}^{\infty}\left\{1-\left|1+d_{n} \beta_{n} / 2\right|^{2}\right\}<\infty$. Now, $\left|1+d_{n} \beta_{n} / 2\right|$ is the absolute value of the expectation $E \exp \left(i y_{n}\right)$ and hence is less than or equal to 1. It follows therefore that $\sum_{1}^{\infty}\left\{1-\left|1+d_{n} \beta_{n} / 2\right|\right\}<\infty$ As $1-$ $\left|1+d_{n} \beta_{n} / 2\right| \geqq \beta_{n} / 2$, this implies that

$$
\sum_{1}^{\infty} \beta_{n}<\infty \text { i.e. } \sum_{1}^{\infty} \operatorname{var} \theta_{n}<\infty \text {. }
$$

From the convergence of $\Pi_{1}^{\infty} E \eta\left(X_{n}\right)$ and $\sum_{1}^{\infty} \beta_{n}$ and the relation $E \eta\left(X_{n}\right)=\left(1+d_{n} \beta_{n} / 2\right) \exp \left(i \alpha_{n}\right)$, we see that $\sum_{1}^{\infty} E \theta_{n}=\sum_{1}^{\infty} \alpha_{n}$ converges. Thus (2.1) implies (2.2).

Lemma 3. A necessary and sufficient condition that $\sum_{1}^{\infty} X_{n}$ exist a.e. is that $\Pi_{1}^{\infty} \eta\left(X_{n}\right)$ exists a.e. for every $\eta \in \hat{G}$, and for some compact neighbourhood $N$ of $e$ 


$$
\sum_{1}^{\infty} P\left(w: X_{n}(w) \notin N\right)<\infty
$$

Proof. Suppose $\sum_{1}^{\infty} X_{n}$ exists a.e. Consequently, for every compact. neighbourhood $N$ of $e, P\left(w: X_{n}(w) \notin N\right.$ i.o. $\left.{ }^{1}\right)=0$ or, equivalently, $\sum_{1}^{\infty} P\left\{w: X_{n}(w) \notin N\right\}<\infty$ by the Borel-Cantelli lemma. That $\Pi_{1}^{\infty} \eta\left(X_{n}\right)$ exists a.e. for each $\eta \in \hat{G}$ follows from the continuity of the characters $\eta$.

Conversely, let $N$ be any compact neighbourhood of $e$ for which (2.3) is satisfied. Since $N-N \supseteqq N$, we have $P\left\{w: X_{n}(w) \notin N-N\right\} \leqq$ $P\left\{w: X_{n}(w) \notin N\right\}$. Hence the symmetric neighbourhood $N-N$ of $e$ also satisfies (2.3). Without loss of generality we may therefore assume that $N$ in (2.3) is symmetric.

Denote by $G^{*}$ the closed subgroup generated by $N$. Necessarily $G^{*}$ is $\sigma$-compact. Further, by Theorem B, $G^{*}$ contains a discrete subgroup $D$ with a finite number of generators such that $G_{1}=G^{*} / D$ is compact and $D \cap(N+N-N)=\{e\}$. Hence by the Borel-Cantelli lemma, (2.3) implies that $P\left\{w: X_{n}(w) \notin N\right.$ i.o. $\}=0$; that is, if $A_{1}=$ $\left\{w: X_{n}(w) \in N\right.$ for all $\left.n \geqq n_{0}(w)\right\}$ then $P\left(A_{1}\right)=1$. Let $\sigma$ be the natural mapping of $G^{*}$ onto $G_{1}$ and write $Y_{n}(w)=\sigma X_{n}(w)$.

As $G_{1}$ is a compact, metric group, $G_{1}$ (and consequently $\hat{G}_{1}$ ) satisfies the second axiom of countablity. Also $\hat{G}_{1}$ is discrete, since $G_{1}$ is compact. Further $\hat{G}_{1}$ consists precisely of those elements of $\hat{G}$ which are identically one on $D$ (cf: Theorem 34 [5]). In view of (2.3), we have $\Pi_{1}^{\infty} \xi\left(Y_{n}\right)$ exists a.e. for each $\xi \in \widehat{G}_{1}$. As $\hat{G}_{1}$ is countable we conclude that, with probability $1, \Pi_{1}^{\infty} \xi\left(Y_{n}\right)$ exists for all $\xi \in \widehat{G}_{1}$. Observe that $G_{1}$, being a compact metric space, is a Baire set of the second category. It is now immediate from Theorem $\mathrm{A}$ that $\sum_{1}^{\infty} Y_{n}$ exists a.e.

Let $A_{2}$ be a set of probability 1 on which $\sum_{1}^{\infty} Y_{n}$ exists. If $A=$ $A_{1} \cap A_{2}$ then $P(A)=1$. Let $w \in A$ and $n \geqq n_{0}(w)$. Hence

$$
X_{n}(w)+X_{n+1}(w) \in N+N \text {. }
$$

As $\sigma(N)$ is a neighbourhood of the identity in $G_{1}$ and since $\sum_{1}^{\infty} Y_{n}(w)$ exists, it is clear that $Y_{n}(w)+Y_{n+1}(w) \in \sigma(N)$, if $n$ is larger than a certain $n_{1}(w)$. That is

$$
X_{n}(w)+X_{n+1}(w) \in N+D \quad \text { if } n \geqq n_{1}(w) .
$$

From (2.4) and (2.5) and the property $D \cap(N+N-N)=\{e\}$, we conclude that $X_{n}(w)+X_{n+1}(w) \in N$ if $n \geqq \max \left(n_{0}, n_{1}\right)$. Repeating the argument a finite number of times it is seen that all finite tails of the series $\sum_{1}^{\infty} X_{n}(w)$ lie in $N$. By exactly similar reasoning, all finite tails lie in any preassigned neighbourhood $M$ of $e$ with $M \subseteq N$. As $N$ is compact, we can show (by arguments similar to the ones

1 infinitely often 
on p. 193 [1]) that $\sum_{1}^{\infty} X_{n}(w)$ exists. Thus on $A$, which is a set of probability $1, \sum_{1}^{\infty} X_{n}$ exists. Combining these results, we have

THEOREM 1. If $\left\{X_{n}, n=1,2, \cdots\right\}$ is an independent sequence of generalised random variables then $\sum_{1}^{\infty} X_{n}$ exists a.e. if and only if the series

(i ) $\sum_{1}^{\infty} P\left\{w: X_{n}(w) \notin N\right\}, N$ being any preassigned compact neighbourhood of $e$,

(ii) $\sum_{1}^{\infty} E \log \eta\left(X_{n}\right)$ and

(iii) $\sum_{1}^{\infty} \operatorname{var} \log \eta\left(X_{n}\right)$ converge for all $\eta \in \hat{G}$. Here $\log \left(X_{n}\right)$ is taken to be $i \theta_{n}$ where $\theta_{n}$ is the principal amplitude of $\eta\left(X_{n}\right)$.

3. Definition. The measure $\mu$ induced in $\mathscr{D}$ by a generalised random variable $f$ will be called the distribution function of $f$. The distribution $\mu$ will be said to be symmetric if $\mu(A)=\mu(-A)$ for every $A \in \mathscr{D}$. It will be called regular if for every $A \in \mathscr{D}, \mu(A)=$ $\sup \{\mu(C): C \subseteq A, C \in \mathscr{C}\}$.

Theorem 2. If $\left\{X_{n}, n=1,2, \cdots\right\}$ is an independent sequence of generalised random variables with regular distributions, then $\sum_{1}^{\infty} X_{n}$ exists a.e. if and only if $\Pi_{1}^{\infty} \eta\left(X_{n}\right)$ exists a.e. for every $\eta \in \widehat{G}$.

Proof. If $\sum_{1}^{\infty} X_{n}$ exists a.e. then $\Pi_{1}^{\infty} \eta\left(X_{n}\right)$ exists a.e. for every $\eta \in \hat{G}$ by the continuity property of $\eta$.

Conversely, let $\Pi_{1}^{\infty} \eta\left(X_{n}\right)$ exist a.e. for each $\eta \in \hat{G}$. The assertion is established through the following steps.

(i) Let $G$ be compact. That the assertion is true in this case is seen by the same reasoning as for $G_{1}$ in Lemma 3.

(ii) Let $G$ be discrete. The compact subsets of $G$ are therefore only those subsets with a finite number of elements. As the distribution of each $X_{n}$ is regular we can find a countable subgroup $G_{1}$ such that $P\left\{w: X_{n}(w) \in G_{1}, n=1,2, \cdots\right\}=1$. Observe that $\hat{G}_{1}$ is the same as $\hat{G}$ restricted to $G_{1}$. Now let the $X_{n}$ 's have symmetric distributions. Hence, if $\varphi_{n}(\eta)=E \eta\left(X_{n}\right)$ then the $\varphi_{n}$ 's are real and $\varphi_{n}(-\eta)=$ $\varphi_{n}(\eta)$. Now by Lemma $1, \Pi_{1}^{\infty} \eta\left(X_{n}\right)$ exists a.e. for each $\eta \in \hat{G}$, implies that $\Pi_{1}^{\infty} \varphi_{n}(\eta)$ exists. Therefore $g(\eta)=\sum_{1}^{\infty}\left\{1-\varphi_{n}(\eta)\right\}$ exists for every $\eta \in \widehat{G}$. If $g_{n}(\eta)=\sum_{1}^{n}\left\{1-\varphi_{k}(\eta)\right\}$ then the $g_{n}$ 's are continuous and $g_{n}(\eta)$ converges monotonically up to $g(\eta)$ as $n \rightarrow \infty$ for each $\eta$. Hence $\{\eta: g(\eta) \leqq a\}=\bigcap_{1}^{\infty}\left\{\eta: g_{n}(\eta) \leqq a\right\}$ is a closed set. $\hat{G}$ is a compact metric space and so is complete. Hence it is a set of the second category. Further, $\hat{G}=\bigcup_{n=1}^{\infty}\{\eta: g(\eta) \leqq n\}$ i.e. $\hat{G}$ is the union of a countable number of closed sets. Therefore by the Baire category theorem, one of these closed sets in the union, say the set $A=\{\eta: g(\eta) \leqq k\}$, has a nonnull interior $V$. Trivially $g$ is bounded on $V$. By the positive 
definiteness and symmetry of $\phi_{k}$,

$$
1-\phi_{k}^{2}(\xi)-\phi_{k}^{2}(\eta)+2 \phi_{k}(\xi) \phi_{k}(\eta) \phi_{k}(\xi+\eta)-\phi_{k}^{2}(\xi+\eta) \geqq 0 .
$$

Let $a_{k}^{2}=1-\dot{\phi}_{k}(\xi), b_{k}^{2}=1-\phi_{k}(\eta)$ and $c_{k}^{2}=1-\phi_{k}(\xi+\eta)$. Then the above inequality implies that

$$
c_{k}^{2} \leqq a_{k}^{2}+b_{k}^{2}-a_{k}^{2} b_{k}^{2}+a_{k} b_{k} \sqrt{\left(2-a_{k}^{2}\right)\left(2-b_{k}^{2}\right)} \leqq\left(a_{k}+b_{k}\right)^{2} .
$$

Consequently,

$$
g(\xi+\eta) \leqq\left\{[g(\xi)]^{1 / 2}+[g(\eta)]^{1 / 2}\right\}^{2}
$$

For any $\xi \in \hat{G}$ consider the open set $\xi-V$. From (3.1) it is immediate that $g$ is bounded on $\xi-V$. The family $\xi-V, \xi \in \hat{G}$ is an open covering for the compact $\hat{G}$. Therefore there exists a finite subcover from this. As $g$ is bounded on each member of this subcover it follows that $g$ is bounded on $\hat{G}$.

Let $m$ be the Haar measure of $\hat{G}$ with $m(\hat{G})=1$. As $P\left\{w: X_{n}(w) \neq e\right\}=$ $\int_{\hat{\theta}}\left\{1-\varphi_{n}(\eta)\right\} d m(\eta)$, we obtain $\sum_{1}^{\infty} P\left(w: X_{n}(w) \neq e\right\}=\int_{\hat{G}} g(\eta) d m(\eta)<\infty$. Since $G$ is discrete this means that for the compact neighbourhood $N=\{e\}$ of $e, \sum_{1}^{\infty} P\left\{w: X_{n}(w) \notin N\right\}<\infty$. That $\sum_{1}^{\infty} X_{n}$ exists a.e. follows from Lemma 3.

(iii) Let $G$ be discrete but the distributions of the $X_{n}$ 's need not be symmetric.

Let $Y_{n}, n=1,2, \cdots$ be another independent sequence of g.r.v.'s and independent of the $X_{n}$ 's; let $Y_{n}$ have the same distribution as $X_{n}, n=1,2, \cdots$.

Write $Z_{n}=X_{n}-Y_{n}$. The $Z_{n}$ 's therefore have symmetric distributions. Also the hypothesis yields that $\prod_{1}^{\infty} \eta\left(Z_{n}\right)$ exists a.e. for every $\eta \in \hat{G}$. Hence by (ii) above

$$
\sum_{1}^{\infty} P\left\{w: Z_{n}(w) \neq e\right\}<\infty .
$$

The distribution of each $X_{n}$ is assumed to be regular. Hence there exists a countable set $A$ such that $P\left\{w: Z_{n}(w) \in A\right.$ for all $\left.n\right\}=1$. Now, if $p_{n}(a)=P\left\{w: X_{n}(w)=a\right\}$, we have

$$
\begin{aligned}
P\left\{w: Z_{n}(w)=e\right\} & =\sum_{a \in A} P\left\{w: X_{n}(w)=a\right\} P\left\{w: Y_{n}(w)=a\right\} . \\
& =\sum_{a \in A} p_{n}^{2}(a) \leqq \sup _{a \in A} p_{n}(a)
\end{aligned}
$$

Since there can only be a finite number of 'values' of $X_{n}$ for which the associated probability is larger than any preassigned number, the supremum is attained. Let $a_{n}$ be any one of the values taken by $X_{n}$ with probability equal to this supremum. Therefore $P\left\{w: X_{n}(w) \neq a_{n}\right\} \leqq$ 
$P\left\{w: Z_{n}(w) \neq e\right\}$. Consequently, using (3.2), we obtain

$$
\begin{array}{cl} 
& \sum_{1}^{\infty} P\left\{w: X_{n}(w) \neq a_{n}\right\}<\infty \\
\text { or } & \sum_{1}^{\infty} P\left\{w: X_{n}(w)-a_{n} \notin N\right\}<\infty .
\end{array}
$$

Where $N$ is the compact neighbourhood of $e$ consisting only of itself. From (3.3) we conclude that, with probability $1, X_{n}=a_{n}$ except for a finite number of $n$ 's. This fact together with the hypothesis implies that $\Pi_{1}^{\infty} \eta\left(a_{n}\right)$ exists for every $\eta \in \hat{G}$. That $\Pi_{1}^{\infty} \eta\left(X_{n}-a_{n}\right)$ exists a.e for every $\eta \in \hat{G}$ is then immediate. Now using (3.4) we see by lemma 3 that $\sum_{1}^{\infty}\left(X_{n}-a_{n}\right)$ exists a.e. By Theorem $\mathrm{A}$ or by applying Lemma 3 to the random variables $a_{n}$ we see however that $\sum_{1}^{\infty} a_{n}$ exists since $\Pi_{1}^{\infty} \eta\left(a_{n}\right)$ exists, for every $\eta \in \hat{G}$. Hence $\sum_{1}^{\infty} X_{n}$ exists a.e., as was to be proved.

(iv) Let $G$ be any metric abelian locally compact group. Let $N$ be a compact symmetric neighbourhood of $e$ and $G^{*}$ the closed subgroup generated by $N$. Necessarily $G^{*}$ is $\sigma$-compact and open. Let $\sigma_{1}$ be the natural mapping of $G$ onto $G_{1}=G / G^{*}$. As $G^{*}$ is open, $G_{1}$ is discrete. Further $\hat{G}_{1}$ consists precisely of those elements of $\widehat{G}$ which are identically one on $G^{*}$. Hence $\Pi_{1}^{\infty} \eta\left(X_{n}\right)$ exists a.e. for each $\eta \in \widehat{G}$ implies that $\Pi_{1}^{\infty} \xi\left(Y_{n}\right)$ exists a.e. for each $\xi \in \widehat{G}_{1}$, where $Y_{n}=\sigma_{1} X_{n}$. By part (iii) above, $P\left\{w: Y_{n}(w) \neq e_{1}\right.$ i.o. $\}=0$ where $e_{1}$ is the identity of $G_{1}$. That is

$$
P\left\{w: X_{n}(w) \notin G^{*}\right\}=0 \text {. }
$$

In other words, there is probability 1 that all except a finite number of the $X_{n}$ 's lie in $G^{*}$.

As $G^{*}$ is generated by a compact symmetric neighbourhood of $e$ there exists, by Theorem $\mathrm{B}$, a discrete group $D$ with a finite number exists, by Theorem $\mathrm{B}$, a discrete group $D$ with a finite number of generators such that $G_{2}=G^{*} / D$ is compact and $D \cap(N-N)=\{e\}$. Let $e_{2}$ be the identity element of $G_{2}$ and $\sigma_{2}$ the natural mapping of $G^{*}$ onto $G_{2}$. Write $Z_{n}=\sigma_{2} X_{n}$ if $X_{n} \in G^{*}$ and $=e_{2}$ if $X_{n} \notin G^{*}$. Hence $Z_{n}, n=1,2, \cdots$ is an independent sequence of g.r.v.'s in $G_{2}$. Recall that $\hat{G}^{*}$ consists of all the elements of $\hat{G}$ restricted to $G^{*}$ and that $\hat{G}_{2}$ consists precisely of those elements of $\hat{G}^{*}$ which are identically 1 on $D$. Using the hypothesis and the equation (3.5) we get $I_{1}^{\infty} \xi\left(Z_{n}\right)$ exists a.e. for every $\xi \in \widehat{G}_{2}$. Therefore we have

$$
P\left\{w: Z_{n}(w) \notin \sigma_{2}(N) \text { i.o. }\right\}=0 \text { i.e. } \quad P\left\{w: X_{n}(w) \notin N+D \text { i.o. }\right\}=0 .
$$

Define $s_{n}=X_{n}$ if $X_{n} \in N+D$ and $s_{n}=e$ if $X_{n} \notin N+D$. Then for each $s_{n}$ we have the unique decomposition $s_{n}=u_{n} \pm v_{n}$ where 
$u_{n} \in N$ and $v_{n} \in D$. The $u_{n}$ 's form an independent sequence of g.r.v.'s and so do the $v_{n}$ 's. It is immediate from the hypothesis that $\Pi_{1}^{\infty} \eta\left(s_{n}\right)$ exists a.e. for each $\eta \in \hat{G}$. Also, since $\Pi_{1}^{\infty} \xi\left(Z_{n}\right)$ exists a.e. for each $\xi \in \widehat{G}_{2}, \Pi_{1}^{\infty} \eta\left(u_{n}\right)$ exists a.e. for each $\eta \in \hat{G}$. Hence $\Pi_{1}^{\infty} \xi\left(v_{n}\right)$ exists a.e. for each $\xi \in \hat{D}$. As $D$ is discrete we have, by part (iii), $P\left\{w: X_{n}(w) \neq\right.$ $e$ i.o. $\}=0$. This is equivalent to saying $P\left\{w: s_{n}(w) \neq u_{n}(w)\right.$ i.o. $\}=0$. Or $P\left\{w: X_{n}(w) \notin N\right.$ i.o. $\}=0$ i.e. $\sum_{1}^{\infty} P\left\{w: X_{n}(w) \notin N\right\}<\infty$. That $\sum_{1}^{\infty} X_{n}$ exists a.e. follows now by Lemma 3 .

I thank the referee for his suggestions leading to a shorter proof of Lemma 1.

\section{REFERENCES}

1. J. L. Kelly, General Topology, New York, D. Van Nostrand Co., 1955.

2. M. Lò̀ve, Probability Theory, New York, D. Van Nostrand Co., 1955.

3. D. Montgomery, and L. Zippin, Topological transformation groups, New York, Interscience Publishers, 1955.

4. B. J. Pettis, On the continuity and openness of homomorphisms in topological groups, Ann. of Math., 51 (1950), 293-308.

5. L. Pontrjagin, Topological Groups, Princeton, 1939.

OSMANIA UNIVERSITY, INDIA

AND

UNIVERSITY OF OREGON 


\title{
PACIFIC JOURNAL OF MATHEMATICS
}

\author{
EDITORS
}

\author{
Ralph S. Phillips \\ Stanford University \\ Stanford, California \\ M. G. Arsove \\ University of Washington \\ Seattle 5 , Washington
}

\author{
J. DugunduI \\ University of Southern California \\ Los Angeles 7, California
}

Lowell J. Paige

University of California

Los Angeles 24, California

\section{ASSOCIATE EDITORS}

E. F. BECKENBACH

T. M. CHERRY
D. DERRY
M. OHTSUKA

H. L. ROYDEN

E. SPANIER
E. G. STRAUS

F. WOLF

\section{SUPPORTING INSTITUTIONS}

UNIVERSITY OF BRITISH COLUMBIA CALIFORNIA INSTITUTE OF TECHNOLOGY UNIVERSITY OF CALIFORNIA MONTANA STATE UNIVERSITY UNIVERSITY OF NEVADA NEW MEXICO STATE UNIVERSITY OREGON STATE UNIVERSITY UNIVERSITY OF OREGON OSAKA UNIVERSITY UNIVERSITY OF SOUTHERN CALIFORNIA
STANFORD UNIVERSITY

UNIVERSITY OF TOKYO

UNIVERSITY OF UTAH

WASHINGTON STATE UNIVERSITY

UNIVERSITY OF WASHINGTON

AMERICAN MATHEMATICAL SOCIETY CALIFORNIA RESEARCH CORPORATION SPACE TECHNOLOGY LABORATORIES NAVAL ORDNANCE TEST STATION

Mathematical papers intended for publication in the Pacific Journal of Mathematics should be typewritten (double spaced), and the author should keep a complete copy. Manuscripts may be sent to any one of the four editors. All other communications to the editors should be addressed to the managing editor, L. J. Paige at the University of California, Los Angeles 24, California.

50 reprints per author of each article are furnished free of charge; additional copies may be obtained at cost in multiples of 50 .

The Pacific Journal of Mathematics is published quarterly, in March, June, September, and December. Effective with Volume 13 the price per volume (4 numbers) is $\$ 18.00$; single issues, $\$ 5.00$. Special price for current issues to individual faculty members of supporting institutions and to individual members of the American Mathematical Society: $\$ 8.00$ per volume; single issues \$2.50. Back numbers are available.

Subscriptions, orders for back numbers, and changes of address should be sent to Pacific Journal of Mathematics, 103 Highland Boulevard, Berkeley 8, California.

Printed at Kokusai Bunken Insatsusha (International Academic Printing Co., Ltd.), No. 6, 2-chome, Fujimi-cho, Chiyoda-ku, Tokyo, Japan.

PUBLISHED BY PACIFIC JOURNAL OF MATHEMATICS, A NON-PROFIT CORPORATION

The Supporting Institutions listed above contribute to the cost of publication of this Journal, but they are not owners or publishers and have no responsibility for its content or policies. 


\section{Pacific Journal of Mathematics}

\section{Vol. 13, No. 2 \\ April, 1963}

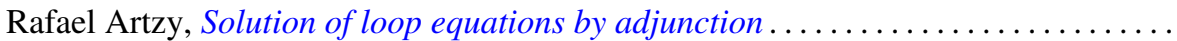

Earl Robert Berkson, A characterization of scalar type operators on reflexive

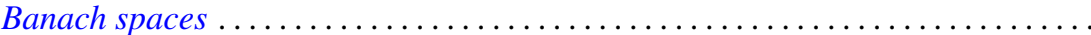

Mario Borelli, Divisorial varieties

365

Raj Chandra Bose, Strongly regular graphs, partial geometries and partially

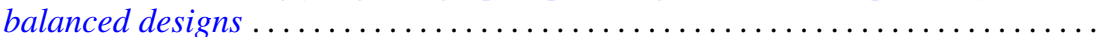

389

R. H. Bruck, Finite nets. II. Uniqueness and imbedding ............... 421

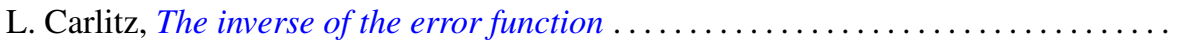

Robert Wayne Carroll, Some degenerate Cauchy problems with operator

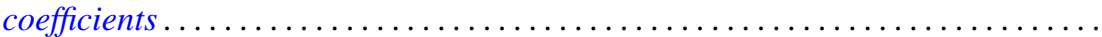

Michael P. Drazin and Emilie Virginia Haynsworth, A theorem on matrices of 0 's

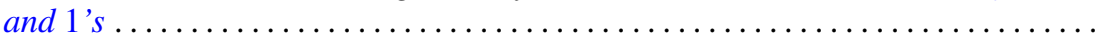

Lawrence Carl Eggan and Eugene A. Maier, On complex approximation .......... James Michael Gardner Fell, Weak containment and Kronecker products of group

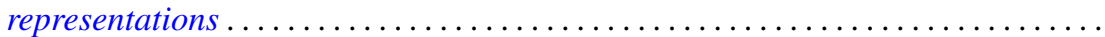

Paul Chase Fife, Schauder estimates under incomplete Hölder continuity

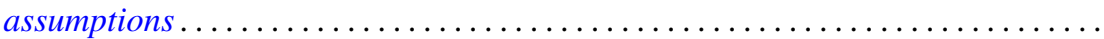

Shaul Foguel, Powers of a contraction in Hilbert space ...................

Neal Eugene Foland, The structure of the orbits and their limit sets in continuous

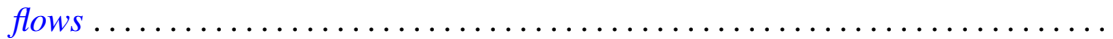

Frank John Forelli, Jr., Analytic measures . . . . . . . . . . . . . . . . . . . . . 563

Robert William Gilmer, Jr., On a classical theorem of Noether in ideal theory ....... P. R. Halmos and Jack E. McLaughlin, Partial isometries .

Albert Emerson Hurd, Maximum modulus algebras and local approximation in

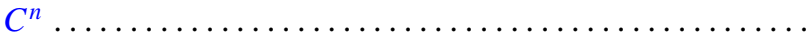

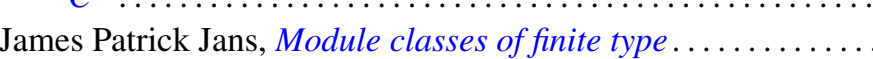

Betty Kvarda, On densities of sets of lattice points ...

H. Larcher, A geometric characterization for a class of discontinuous groups of linear fractional transformations .

John W. Moon and Leo Moser, Simple paths on polyhedra .

T. S. Motzkin and Ernst Gabor Straus, Representation of a point of a set as sum of

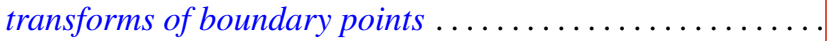

Rajakularaman Ponnuswami Pakshirajan, An analogue of Kolmogorov's three-series theorem for abstract random variables ...............

Robert Ralph Phelps, Čebyšev subspaces of finite codimension in $C(X)$...

James Dolan Reid, On subgroups of an Abelian group maximal disjoint from a given subgroup ...

William T. Reid, Riccati matrix differential equations and non-oscillation criteria for associated linear differential systems .................

Georg Johann Rieger, Some theorems on prime ideals in algebraic number fields ...

Gene Fuerst Rose and Joseph Silbert Ullian, Approximations of functions on the

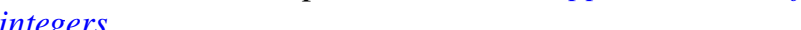

F. J. Sansone, Combinatorial functions and regressive isols . . . . . . . . . . 703

Leo Sario, On locally meromorphic functions with single-valued moduli . . . . . . . 709

Takayuki Tamura, Semigroups and their subsemigroup lattices.

Pui-kei Wong, Existence and asymptotic behavior of proper solutions of a class of second-order nonlinear differential equations . . ........... 